\title{
Effect of siRNAs targeting the EGFR T790M mutation in a non-small cell lung cancer cell line resistant to EGFR tyrosine kinase inhibitors and combination with various agents
}

\author{
Gang Chen ${ }^{\mathrm{a}, \mathrm{b}}$, Peter Kronenberger ${ }^{\mathrm{a}, \mathrm{c}}$, Erik Teugels ${ }^{\mathrm{a}}$, Ijeoma Adaku Umelo ${ }^{\mathrm{a}}$, Jacques De Grève ${ }^{\mathrm{a}, *}$

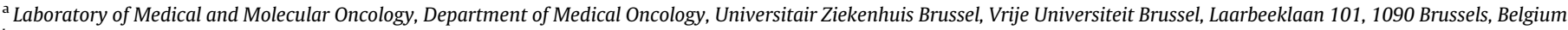 \\ ${ }^{\mathrm{b}}$ Department of Pathology, First Affiliated Hospital, Guangxi Medical University, Shuangyong Road 6, 530021 Nanning, Guangxi, PR China \\ ${ }^{\mathrm{c}}$ Laboratory for Biotechnology, Department of Healthcare, Erasmushogeschool Brussel, Laarbeeklaan 121, 1090 Brussels, Belgium
}

\section{A R T I C L E I N F O}

\section{Article history:}

Received 11 December 2012

Available online 22 December 2012

\section{Keywords:}

T790M

EGFR

Non-small cell lung cancer

RNA interference

Afatinib

Cetuximab

\begin{abstract}
A B S T R A C T
The epidermal growth factor receptor (EGFR) is a validated therapeutic target in non-small cell lung cancer (NSCLC). However, some mutations confer resistance to current available agents, especially the frequently occurring T790M mutation. In the current study, we have examined, in a NSCLC cell line H1975 containing both L858R and T790M mutations, the effect of T790M-specific-siRNAs versus other EGFR-specific-siRNAs. T790M-specific-siRNAs were able to inhibit T790M and EGFR mRNA, to reduce EGFR protein expression, as well as to reduce the cell growth and induce cell caspase activity in H1975 cells. However, this effect showed less potency compared to the other EGFR-specific-siRNAs. EGFR-specific-siRNAs strongly inhibited cell growth and induced apoptosis in H358, H1650, H292, HCC827 and also in H1975 cells, which showed weak response to tyrosine kinase inhibitors (TKIs) or cetuximab. The addition of T790M-specific-siRNAs could rescue the sensitivity of T790M mutant H1975 cells to TKIs. The combination of T790M-specific-siRNAs and cetuximab also additively enhanced cell growth inhibition and induction of apoptosis in H1975 cells. Among the anti-EGFR agents tested, the strongest biological effect was observed when afatinib was combined with T790M-specific-siRNAs. Afatinib also offered extra effect when combined with cetuximab in $\mathrm{H} 1975$ cells. In conclusion, knock-down of T790M transcript by siRNAs further decreases the cell growth of T790M mutant lung cancer cells that are treated with TKIs or cetuximab. The combination of a potent, irreversible kinase inhibitor such as afatinib, with T790M-specific-siRNAs should be further investigated as a new strategy in the treatment of lung cancer containing the resistant T790M mutation.
\end{abstract}

(c) 2012 Elsevier Inc. All rights reserved.

\section{Background}

Lung cancer is one of the most frequent neoplasm worldwide and about $85-90 \%$ of lung cancer is non-small cell lung cancer (NSCLC) [1]. Activating mutations in the kinase domain of epidermal growth factor receptor (EGFR) in NSCLC commonly arise as in-frame deletions in exon 19 and L858R exon 21 base substitution, and confer sensitivity to the reversible tyrosine kinase inhibitors (TKIs) [2,3]. Despite initial responses, NSCLCs driven by EGFR activating mutations inevitably develop resistance to these TKIs. An acquired substitution mutation (T790M) emerges in approximately $50 \%$ of EGFR-mutated patients with TKI resistance [4]. The threonine to methionine change at the 790 amino acid "gatekeeper" residue in the EGFR kinase domain has been shown to confer resistance by increasing the affinity for ATP, compromising the po-

\footnotetext{
* Corresponding author. Address: Oncology Center, UZ Brussel, Vrije Universiteit Brussel (VUB), Laarbeeklaan 101, 1090 Brussels, Belgium. Fax: +32 24776210.

E-mail address: Jacques.Degreve@uzbrussel.be (J. De Grève).
}

tency of reversible TKIs [5]. In these T790M-harboring cells, inhibition of EGFR by currently available second-generation EGFR-TKIs is still not sufficient to physiologically prevent the emergence of cells that are dependent on EGFR signaling [6]. For instance, afatinib (BIBW 2992, Boehringer Ingelheim GmbH), a panHER inhibitor of EGFR, HER2 and HER4 kinases, retains some activity in tumors with a T790M mutation. However, afatinib showed limited efficacy in NSCLC with T790M [7]. Therefore, strategies to overcome TKI resistance remain practical needs in order to prolong survival of patients with NSCLC. RNA interference (RNAi) has provided a powerful tool with which to modulate gene expression for the study of gene function. Several reports described effects of EGFRtargeted RNAi to inhibit cell growth [3,4,8-10], however attempts to knock-down the T790M-containing allele (using shRNA constructs) were unsuccessful [4]. In the current study we have for the first time investigated the combined effect of RNAi targeting T790M mRNA with T790M-specific-siRNAs, and inactivation of EGFR signaling using different TKIs or the monoclonal antibody cetuximab in the cell line H1975 that carries the T790M mutation. 


\section{Material and methods}

\section{1. siRNAs, cell lines and reagents}

Eight siRNAs out of 21 candidate sequences containing the T790M mutation were selected by applying algorithms from Maurice HO Rational siRNA design (http://ihome.ust.hk/ bokcmho/siRNA/siRNA.html), Dharmacon (http://www.dharmacon.com), Reynolds [11], Ui-Tei [12] and Jagla [13]. Eight siRNAs targeting wild type (wt) EGFR sequences were designed using algorithms from Invitrogen, Eurogentec, or Dharmacon or were modified from the literature (Table 1). All siRNAs were purchased from Eurogentec (Liege, Belgium). The glyceraldehyde-3-phosphate dehydrogenase (GAPDH) positive control siRNA (used in preliminary experiments to optimize transfection efficiency, data not shown) was from Invitrogen (Merelbeke, Belgium). The negative control siRNA was a proprietary sequence that does not correspond to any eukaryotic gene (Liege, Belgium). The siRNA duplexes with the final concentration $200 \mathrm{nM}$ (for siRNA pool, $50 \mathrm{nM}$ of each individual siRNA were used) were transiently transfected using lipofectamine $^{\mathrm{TM}} 2000$ as described previously [14]. The information about the cell lines and preparation for different anti-EGFR agents were described previously [14]. Each experiment was performed at least in triplicate and three times independently.

\subsection{RT-qPCR and Western blot analysis}

RNA isolation, normalization, real time RT-qPCR for T790M, EGFR and GAPDH and western blot were as described previously [14-16]. The following primary antibodies were used: EGFR (Cell
Signaling), phospho-EGFR (Tyr1173, clone 9H2, Upstate), phospho-AKT/PKB (pS473, Invitrogen), phospho-ERK1/2 (pTpY185/ 187, Invitrogen), phospho-STAT3 (Tyr705, 3E2, Cell Signaling), phospho-STAT5 (pY694, BD Biosciences) and $\beta$-actin (Sigma-Aldrich N.V.).

\subsection{Cell functional detections}

Cell growth was assessed using a colorimetric tetrazolium assay (substrate MTS, CellTiter96 AQueous One Solution Cell Proliferation Assay, Promega, Madison, USA). For the siRNAs + TKI/antibody combinations, siRNA transfection was performed first, and $24 \mathrm{~h}$ later the cells were treated with different anti-EGFR agents in the same wells and incubated for another $72 \mathrm{~h}$. Cell viability was detected by fluorimetric detection of resorufin (CellTiter-Blue Cell Viability Assay, Promega, Madison, USA). Caspase-3/7 activity was measured using a synthetic rhodamine labeled caspase-3/7 substrate performed immediately after the detection of viability on the same wells. The effects on apoptosis and nuclear morphology in the cells were assessed by Hoechst 33342 and propidium iodide (PI, Sigma-Aldrich N.V. Bornem, Belgium) double fluorescent chromatin staining. The protocols were as described previously [14].

\subsection{Statistical analysis}

SPSS19.0 was used for statistical analysis. Values were presented as the mean \pm standard deviation (SD). One-way Analysis of Variance (ANOVA) test was performed to analyze significance between groups. The Least Significant Difference (LSD) method of

Table 1

EGFR siRNAs used in the study.

\begin{tabular}{|c|c|c|c|c|c|}
\hline Name of siRNA & Exon & Sequence & Location and length (nt) & Designed by & GC content $(\%)$ \\
\hline EGFR siRNA486 & $2-3$ & GACCATCCAGGAGGTGGCTGGTTAT & $486-510(25)$ & Invitrogen & 56.0 \\
\hline EGFR siRNA604 & 3 & GCAGTCTTATCTAACTATGATGCAA & $604-628(25)$ & Invitrogen & 36.0 \\
\hline EGFR siRNA752 & 4 & GCAGTGACTTTCTCAGCAA & $752-770(19)$ & Eurogentec & 47.4 \\
\hline EGFR siRNA1247 & $8-9$ & GCAAAGTGTGTAACGGAATAGGTAT & $1247-1271(25)$ & Invitrogen & 40.0 \\
\hline EGFR siRNA1608 & 12 & GGAGATAAGTGATGGAGAT & $1608-1626(19)$ & Eurogentec & 42.1 \\
\hline EGFR siRNA2654 & 20 & GGGAACACAAAGACAATAT & $2654-2672(19)$ & Dharmacon & 36.8 \\
\hline EGFR siRNA2708 & $20-21$ & TCGCAAAGGGCATGAACTA & $2708-2726(19)$ & Dharmacon & 47.4 \\
\hline EGFR siRNA4765 & 28 & AGAATGTGGAATACCTAAGG & $4766-4785(20)$ & $*$ & 40.0 \\
\hline Scrambled EGFR siRNA486 & & GAGGTTAGCTGGGTCCCGATCATGA & $\mathrm{NA}(25)$ & is & 56.0 \\
\hline Scrambled EGFR siRNA604 & & GCATTGTCATTAAACGCAACGTATT & $\mathrm{NA}(25)$ & $\dot{s}$ & 36.0 \\
\hline Scrambled EGFR siRNA752 & & GGCTAAGCTCGCTATTACA & $\mathrm{NA}(19)$ & is & 47.4 \\
\hline Scrambled EGFR siRNA1247 & & GGTGATTAGGTTATAAACGGACAGA & $\mathrm{NA}(25)$ & is & 40.0 \\
\hline T790M siRNA2596 & 20 & TCCACCGTGCAGCTCATCATG & $2596-2616(21)$ & $\triangle$ & 57.1 \\
\hline T790M siRNA2597 & 20 & CCACCGTGCAGCTCATCATGC & $2597-2617(21)$ & $\triangle$ & 61.9 \\
\hline T790M siRNA2600 & 20 & CCGTGCAGCTCATCATGCAGC & $2600-2620(21)$ & $\triangle$ & 61.9 \\
\hline T790M siRNA2601 & 20 & CGTGCAGCTCATCATGCAGCT & $2601-2621(21)$ & $\triangle$ & 57.1 \\
\hline T790M siRNA2603 & 20 & TGCAGCTCATCATGCAGCTCA & $2603-2623(21)$ & $\triangle$ & 52.4 \\
\hline T790M siRNA2607 & 20 & AGCTCATCATGCAGCTCATGC & $2607-2627(21)$ & $\triangle$ & 52.4 \\
\hline T790M SiRNA2608 & 20 & GCTCATCATGCAGCTCATGCC & $2608-2628(21)$ & $\triangle$ & 57.1 \\
\hline T790M siRNA2612 & 20 & TCATGCAGCTCATGCCCTTCG & $2612-2632(21)$ & $\triangle$ & 57.1 \\
\hline Scrambled T790MsiRNA2597 & & GCTCAGCGACGCCTCATACCT & $\mathrm{NA}(21)$ & 访 & 61.9 \\
\hline Scrambled T790MsiRNA2600 & & GCGCAGTCGCTAGCTCACTCA & $\mathrm{NA}(21)$ & is & 61.9 \\
\hline Scrambled T790MsiRNA2603 & & GCATTAGGCGTCCCACTCAAT & $\mathrm{NA}(21)$ & is & 52.4 \\
\hline Scrambled T790MsiRNA2608 & & GCCTCTAGTATCCGGACACCT & $\mathrm{NA}(21)$ & $i$ & 57.1 \\
\hline \multicolumn{6}{|l|}{ Wt for T790MsiRNA2597, } \\
\hline EGFRwtsiRNA2597 & 20 & CCACCGTGCAGCTCATCACGC & $2597-2617(21)$ & NA & 66.7 \\
\hline \multicolumn{6}{|l|}{ Wt for T790MsiRNA2600, } \\
\hline EGFRwtsiRNA2600 & 20 & CCGTGCAGCTCATCACGCAGC & $2600-2620(21)$ & NA & 66.7 \\
\hline Wt for T790MsiRNA2603, EGFRwtRNA2603 & 20 & TGCAGCTCATCACGCAGCTCA & $2603-2623(21)$ & NA & 57.1 \\
\hline Wt for T790MsiRNA2608, EGFRwtRNA2608 & 20 & GCTCATCACGCAGCTCATGCC & $2608-2628(21)$ & NA & 61.9 \\
\hline
\end{tabular}

*, Modified from Ref. [4].

$\triangle$, Different siRNA design algorithms.

放, By http://www.sirnawizard.com/scrambled.php.

NA, not applicable. 
multiple comparisons was applied when the probability for ANOVA was statistically significant. Statistical significance was determined at a $P<0.05$ level. In the analysis of additivity and synergism, the theoretical zero-interaction (exactly additive) dose-response curve for each siRNA + drug combination was calculated by applying the Bliss independence criterion [17]. Determination of possible synergy was also assessed by the Biosoft CalcuSyn program (Ferguson, MO, USA). The Combination Index (CI) was used to express synergism $(\mathrm{CI}<1)$, additive effect $(\mathrm{CI}=1)$, or antagonism $(\mathrm{CI}>1)[18]$.

\section{Results}

\subsection{Effect of T790M and EGFR-specific-siRNAs on target expression and malignant phenotype}

The effect of eight siRNAs targeting T790M with the highest scores obtained from different siRNA designing software [11-13] was firstly investigated. All eight T790M-specific-siRNAs could downregulate T790M mRNA levels (data not shown) and reduce cell growth (Supplementary Fig. 1A) with variable efficacy in H1975 cells, whereas the negative siRNA control and scrambled controls produced little or no effect (Supplementary Fig. 1B). The most effective siRNAs targeting T790M (T790MsiRNA2597, 2600, 2603,2608 ) were selected to form a pool for subsequent experiments. Likewise, eight individual EGFR-specific-siRNAs corresponding to different exons were designed or modified. Among these eight different EGFR-specific-siRNAs that were assessed for their ability to reduce EGFR mRNA levels and inhibit cell proliferation in different NSCLC cell lines, four were chosen to compose a siRNA pool (EGFRsiRNA486, 604, 752, 1247).
In H1975 cells, siRNAs targeting the T790M mutation were able to knock-down the T790M transcript (from 76\% to $85 \%$ ), as detected $96 \mathrm{~h}$ post-transfection by the transcript-specific RT-qPCR assay which we set up previously. This assay allows to detect as little as $1 \%$ mutant transcripts in a wt background [15]. The T790M siRNA pool offered no extra knock-down effect with the knock-down ratio of $79 \pm 2 \%$. The siRNAs targeting wt sequences were able to knock-down the EGFR transcript in different NSCLC cell lines. The EGFR siRNA pool was more potent to downregulate the EGFR mRNA levels (data not shown). The EGFR-specific-siRNAs also affected T790M transcript levels in H1975 cells, although differentially and much weaker than the effect of EGFR-specific-siRNAs knocking-down of wt EGFR mRNA or T790M-specific-siRNAs downregulating T790M transcripts (data not shown).

EGFR protein expression in different cell lines transfected with EGFR-specific-siRNAs was severely reduced. The EGFR-specific-siRNA pool downregulated the EGFR protein level more potently than single siRNA (data not shown). The knock-down achieved almost the same magnitude in all cell lines studied, and was independent on the presence or absence of the T790M mutation. The siRNAs targeting T790M could also down-regulate the EGFR protein expression slightly in H1975 cells, but less than the wt EGFR-specific siRNAs (Fig. 1A).

A colorimetric MTS tetrazolium assay revealed that there was a time and dose dependent reduction of up to $25 \%$ of cell growth in H1975 cells with T790M-specific-siRNAs. Consistent with the mRNA level, the effect of T790M-specific-siRNA pool was equivalent to the single siRNA (Fig. 1B and C). The T790M-specific-siRNAs had little influence on the cell proliferation of other NSCLC cell lines (H358, H1650, H292, HCC827, Supplementary Fig. 2). The inhibition ratio of cell growth caused by single EGFR-specific-siRNAs in H1975 cells could reach 30\%. The inhibition effect was much
A
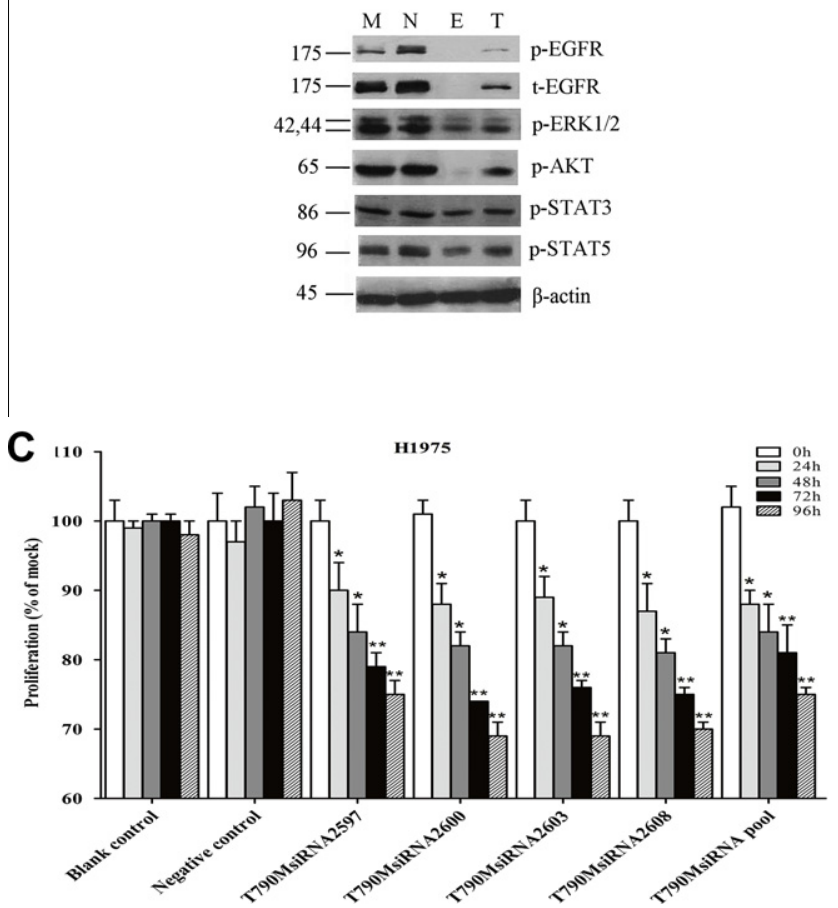

B
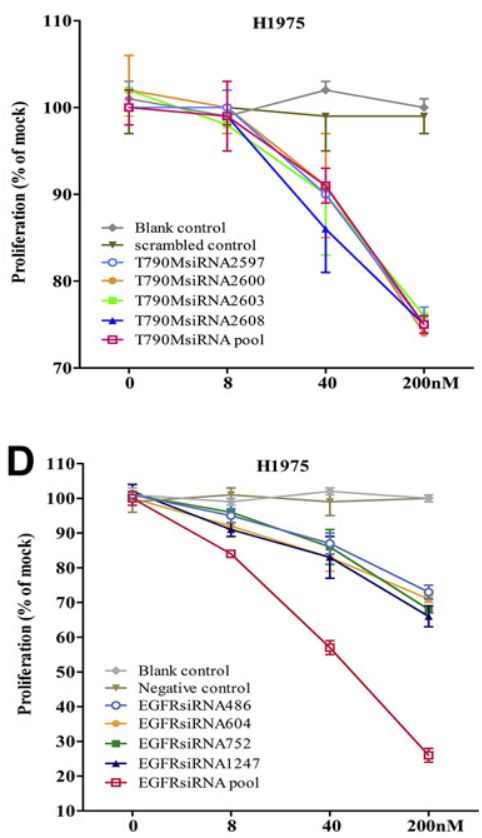

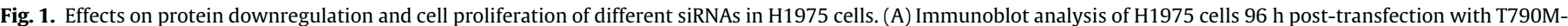

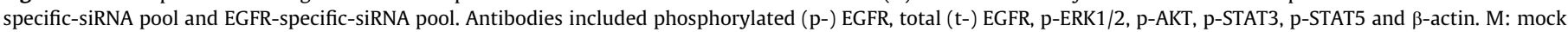

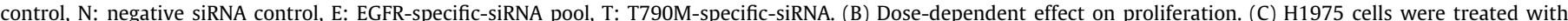

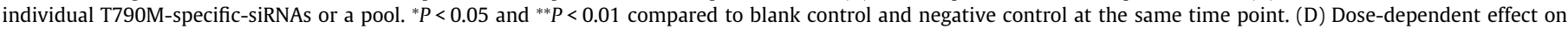
proliferation with EGFR-specific-siRNAs or a pool. 
stronger by the EGFR-specific-siRNA pool, which was even more than two-fold stronger compared to the single siRNA (Figs. 1D and $2 \mathrm{~A}$ ). To verify these results, the effect on viability was assessed using a fluorimetric resorufin viability assay and by microscopic counting of viable (Hoechst 33342 positive/PI negative) cells (Figs. 2B and 3). In both assays the results largely mirrored the MTS tetrazolium assay results. EGFR siRNA pool also yielded a stronger effect on cell growth suppression in the cell lines without T790M mutations compared to single siRNA (Supplementary Fig. 3).

Of particular interest is the capacity of these siRNAs to induce apoptosis. The tetrazolium assay was multiplexed with a fluorescent caspase-3/7 assay. The results show a dose dependent increase of the caspase-3/7 signal in H1975 cells with all T790Mspecific-siRNAs. The effect of the T790M-specific-siRNA pool was slightly stronger than the single siRNA (Fig. 2C). A time dependent manner was also observed to induce caspase-3/7 activity in H1975 cells with T790M-specific-siRNAs (Fig. 2D). The effect of caspase induction was nearly three-fold higher compared to mock control 96 h post-transfection (Fig. 2C and D). This activity was confirmed by microscopic counting of apoptotic cells with Hoechst 33342 and PI staining (Fig. 3). The T790M-specific-siRNAs had little effect on the caspase activity induction in H358, H1650, H292 and HCC827 cells. The EGFR-specific-siRNA pool induced more caspase signals compared to T790M-specific-siRNA pool in H1975 cells (Fig. 2D). The EGFR-specific-siRNA pool also induced caspase-3/7 activity in other 4 cell lines with different magnitude. The least sensitive cell line was the H292 cell line (EGFR wt) in which even at the highest concentration tested and longest time, a three-fold increase in apoptotic rate was not obtained, while in the other cell lines a three-fold apoptotic rate was reached within $72 \mathrm{~h}$ (Supple- mentary Fig. 4). The T790M-specific-siRNAs in H1975 cells affect slightly the downstream pathways: ERK, AKT and STAT. The downregulation of these pathways by EGFR-specific-siRNA was however much more effective, especially the AKT pathway (Fig. 1A). This might explain the weaker effect observed with T790M-specificsiRNA on cell growth inhibition and apoptosis induction compared to EGFR-specific-siRNA treatment in the H1975 cells.

\subsection{Effect of adding T790M-specific-siRNAs to either EGFR TKIs or to cetuximab}

H1975 cells were treated with reversible EGFR TKIs gefitinib and erlotinib, the panHER inhibitor afatinib, or with the monoclonal antibody cetuximab. Afatinib produced a stronger inhibition (IC50 $=0.9827 \pm 0.1784 \mu \mathrm{M})$ on cell growth than the reversible kinase inhibitors gefitinib (IC50 $=5.4405 \pm 0.3394 \mu \mathrm{M})$ and erlotinib (IC50 $=6.7519 \pm 0.2507 \mu \mathrm{M})$, or the monoclonal antibody cetuximab $($ IC50 $=2.7994 \pm 0.2285 \mu \mathrm{M})$, as assessed by MTS assay. The effect of the anti-EGFR agents was also studied using the fluorimetric resorufin viability assay, yielding analogous results (data not shown). The combination of T790M-specific-siRNAs with TKIs or cetuximab on cell growth in H1975 cells was also studied using the MTS assay. There was an enhancement of cell growth inhibition in H1975 cells treated with the T790M-specific-siRNAs + drug combinations compared to single agent. The most potent combination was the T790M-specific-siRNAs plus afatinib (Fig. 4). As can be seen in Fig. 4, addition of T790M-specific-siRNAs systematically further reduced cell growth over afatinib alone. Conversely, by comparing also zero afatinib dose with the samples treated with afatinib in increasing doses it is also apparent that the addition of afatinib to siRNA also increases the growth inhibition.
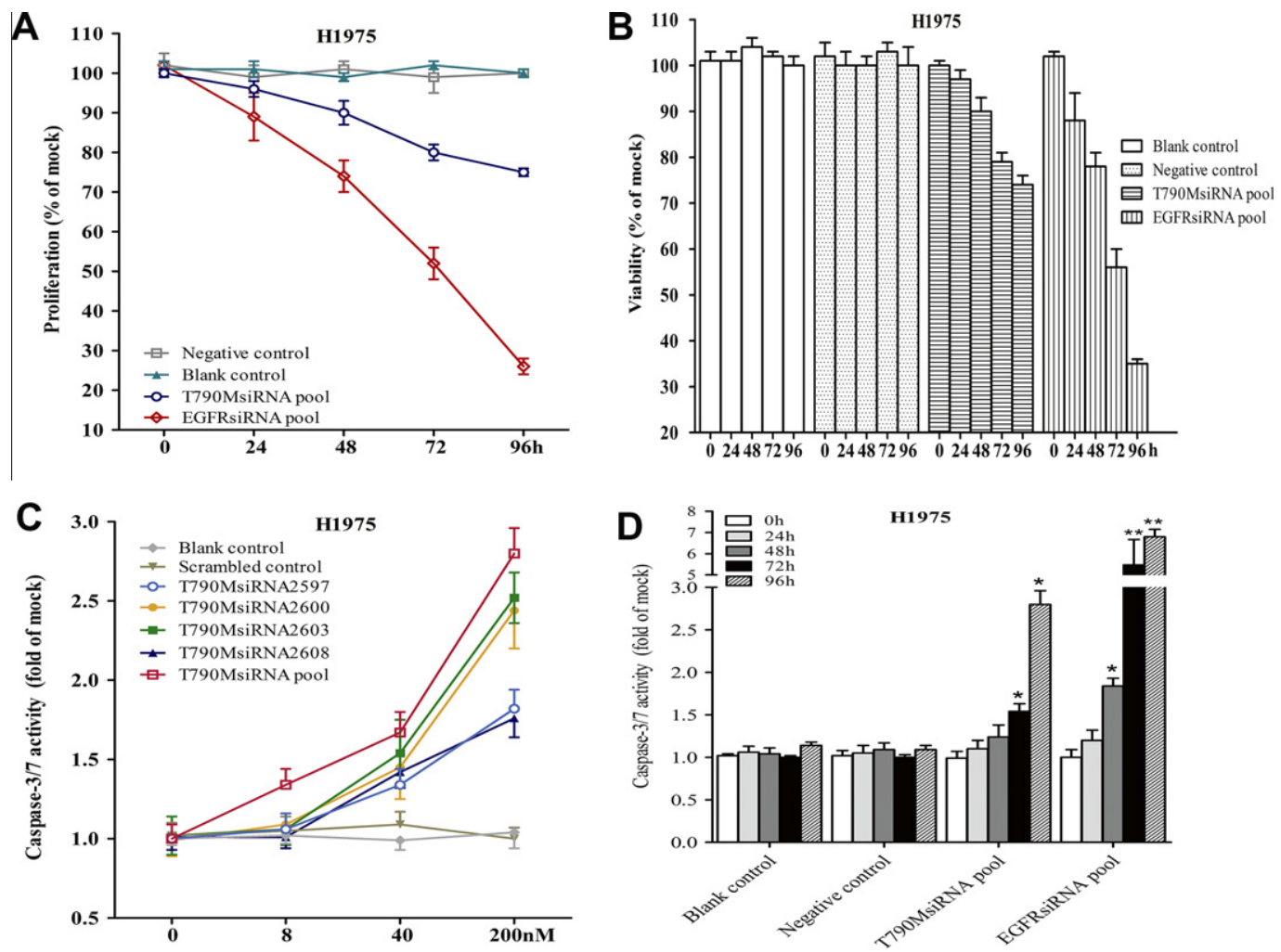

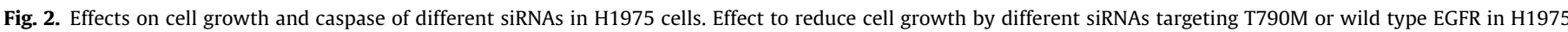

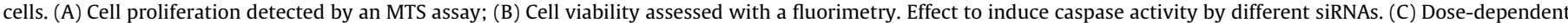

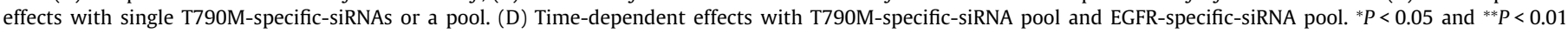
compared to blank control and negative control at the same time point. 


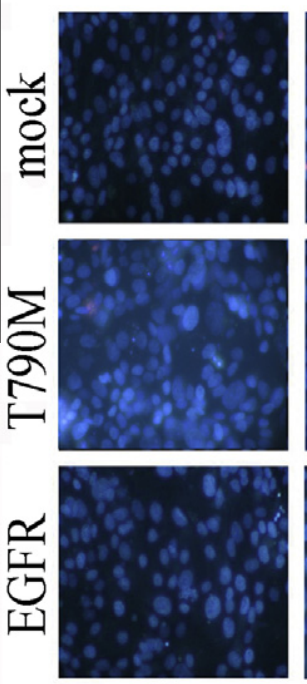

$\mathrm{Oh}$
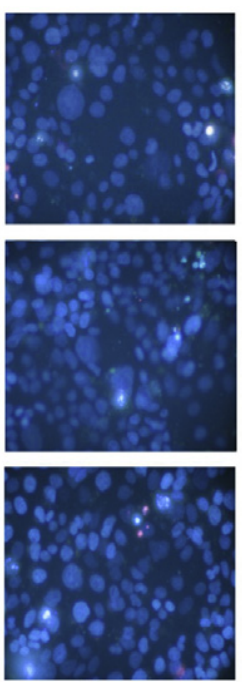

$24 \mathrm{~h}$

\section{H1975}
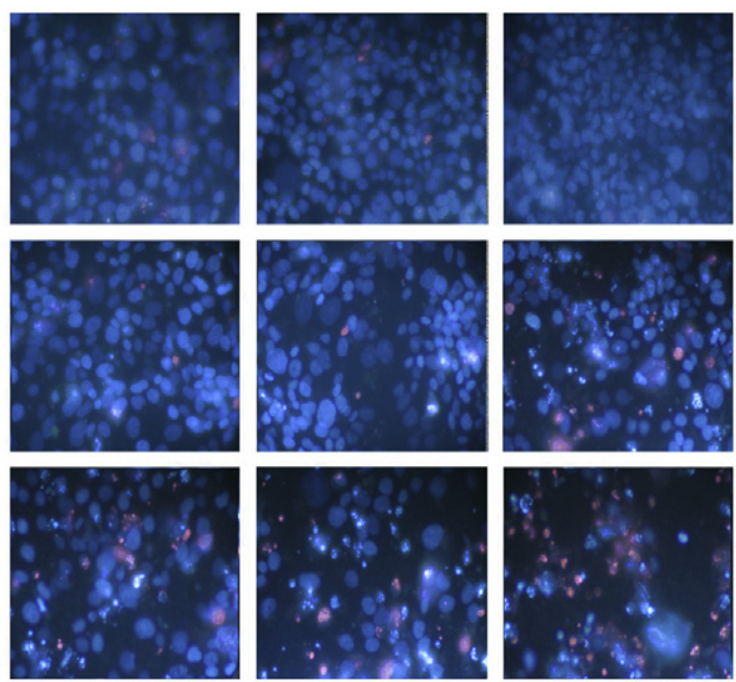

$48 \mathrm{~h}$

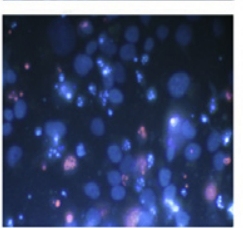

$72 \mathrm{~h}$

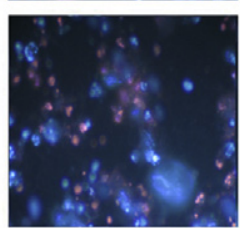

$96 \mathrm{~h}$

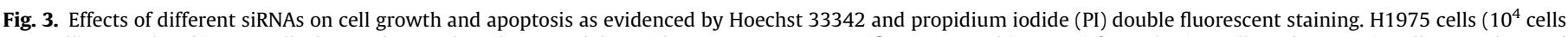

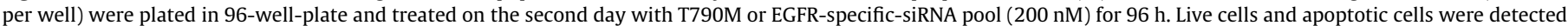
with Hoechst 33342 and PI double fluorescent staining. The number of apoptotic cells was normalized to the number of live cells in the same well $200 \times$.
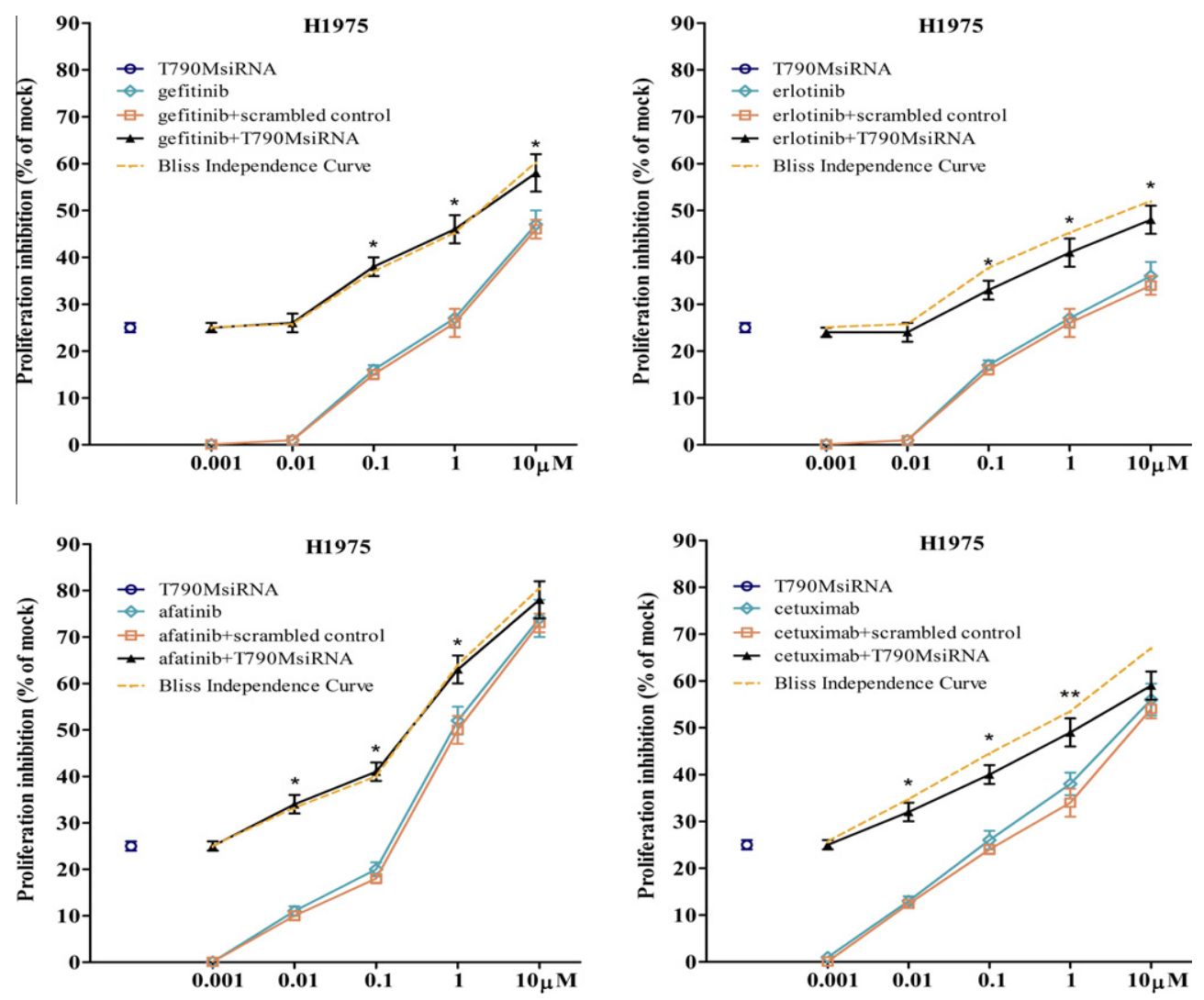

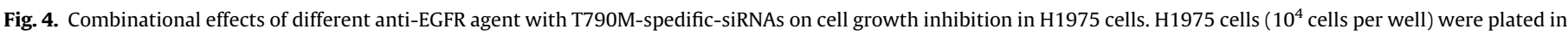

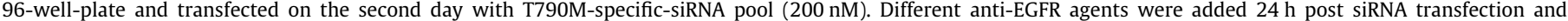
incubated for another 72 h. ${ }^{*} P<0.05$ and ${ }^{* *} P<0.01$, compared to each agent alone.

To ascertain the additive or synergistic nature, a combination index was calculated [17]. The results unambiguously show that the combined inhibition of proliferation is additive for all the combinations, since the combination indexes are close to or equal to one (data not shown). There was also a potentiation of apoptosis in H1975 cells treated with the T790M-specific-siRNAs + drug combinations versus single agent alone (Supplementary Fig. 5). The combinational effect was only clearly observed at doses of $10 \mu \mathrm{M}$ of 
erlotinib and cetuximab, and between 1 and $10 \mu \mathrm{M}$ of gefitinib. The strongest combinational activity was observed at supra micro molar doses of afatinib. The combination of afatinib and cetuximab has been reported to overcome resistance due to T790M mutation both in vivo [19] as well as clinically [20]. We then tested the combinational effect of afatinib and cetuximab on $\mathrm{H} 1975$ cells in vitro. The addition of afatinib further enhanced the effect of cetuximab to inhibit cell growth. However, the effect was additive, as analyzed by the combination index (Supplementary Figs. 6 and 7A). Meanwhile, afatinib increased the effect of cetuximab to induce apoptosis (Supplementary Fig. 7B).

\section{Discussion}

In a previous study we have shown that the addition of EGFRspecific-siRNA to TKIs or cetuximab increases the therapeutic effects in different NSCLC cell lines with various genomic background [14]. Besides that, only a few publications report on the biological effects of RNAi targeting EGFR on lung cancer cells $[3,4,8-10]$. In the present study, we further enhanced the effect of EGFR-specific-siRNAs by introducing a siRNA pool consisting of four individual siRNAs. The EGFR-specific-siRNA pool led to a much more potent activity in downregulating EGFR mRNA, and protein, as well as in reducing cell growth and inducing cell apoptosis, compared to single siRNA, regardless of driver gene background in different cell lines.

At least half of the acquired resistance to EGFR-TKIs in advanced NSCLC patients is considered to be mediated by the "gatekeeper" mutation T790M [14]. Specifically targeting this T790M mutation, using RNAi, has been studied once. Engelman et al. [4] generated six shRNA constructs to specifically downregulate the T790M-containing allele. None of the constructs was found to effectively downregulate the EGFR (shRNA sequences were however not specified). In the present study, siRNAs specifically targeting the T790M mutation decreased cell growth and induced apoptosis signals to various degrees. They also modestly downregulated downstream signaling (p-AKT, p-ERK1/2 and p-STAT5) in H1975 cells, but much weaker compared to EGFR wt siRNAs. Effects were also detectable at the mRNA level, by real-time RT-qPCR. With a T790M-specificsiRNA pool, approximately 80\% T790M mRNA knock-down was obtained, based on our transcript-specific real time RT-qPCR assay. However, in contrast to what was observed with the EGFR-specific-siRNA pool, the effect of the T790M-specific-siRNA pool was not superior compared to single siRNA. The discrepancy between T790M-specific-siRNA pool and EGFR-specific-siRNA pool could be explained by the different range over the EGFR sequence covered by these two siRNA pools. The T790M-specific-siRNA pool includes T790MsiRNA2597, 2600, 2603 and 2608, which covers only 31 bp length (from 2597th to 2628th). Whereas the EGFR-specificsiRNA pool covers 786 bp from exon 2 to exon 9 (from 486th to 1271th). None of the T790M-specific-siRNAs tested here favored knock-down of T790M over wt EGFR transcript. However, the T790M-specific-siRNAs reduced cell growth and induced cellular apoptosis in H1975 cells more modestly compared to EGFR-specific-siRNAs. However, the T790M-specific-siRNAs did have a selective effect against T790M versus other mutant forms of EGFR or wt EGFR, leading to specific funtional effects in the cell line carrying this mutation. This could have advantages for in vivo application with regard to toxicity.

Anti-EGFR TKIs and antibodies differ in action mechanisms. However, none of these agents alone does maximally suppress EGFR signaling, as also shown in our experiments. The combination of afatinib and cetuximab has been reported to overcome T790M resistance both preclinically [19] and clinically [20]. Regales et al. [19] found that dual targeting of EGFR with afatinib and cetuximab could induce regression of H1975 cell xenografts and thus over- come the major drug resistant T790M mutation. A clinical trial of combination of afatinib and cetuximab on NSCLC is now ongoing (ClinicalTrials.gov Identifier: NCT01090011, sponsor: Boehringer Ingelheim Pharmaceuticals, http://www.clinicaltrials.gov). In the current study, we tested the combinatorial effect of afatinib and cetuximab on H1975 cells. The combination of these agents had a stronger effect on cell growth inhibition and induction of caspase activity. However, the effect is additive, not synergistic. The less potent effect in vitro from the combination compared to that in vivo could be due to the micro-environmental conditions in vivo. The complement mediated tumor cell killing (CDC) or antibody-dependent cell-mediated cytotoxicity (ADCC) could play a vital role in the in vivo effect of cetuximab. Preclinical results have already shown that cetuximab has the ability of inducing either CDC or ADCC by interacting with natural killers (NKs), monocytes and granulocytes by means of their specific Fc receptors [21]. The lack of host responses influences the effect of cetuximab, thus reduces the combinational outcome of cetuximab and afatinib.

Since the T790M mutation is a main cause of the acquired resistance to first generation TKIs, in the present study, we combined the T790M-specific-siRNAs with different anti-EGFR agents. The combined treatment of T790M-specific-siRNAs and TKIs or antibody in H1975 achieved increased tumor cell growth suppression (max. about 30\%, Fig. 4) and increased apoptosis by more than two-fold (Supplementary Fig. 5). The effect with the different agents in the different cell lines was additive. Thus, when T790M is eliminated by RNAi, the sensitivity to TKIs is partially rescued. The differential sensitivity to the combination paralleled the differential sensitivities to the TKIs tested or cetuximab alone. Afatinib with T790M-specific-siRNAs achieved the strongest combinatorial effect. Even at very low concentration of afatinib (10-100 nM), the dual effect on cell growth inhibition and caspase activity induction was significantly more potent compared to the single treatment. The combination of gefitinib, erlotinib or cetuximab with T790Mspecific-siRNAs had less potent activity.

We conclude that RNA interference by siRNA oligonucleotides targeting the T790M mutation should be further explored and developed as a therapeutic modality in the treatment of T790M mutant lung cancer. The selectivity of these siRNAs for the T790M mutant EGFR as opposed to other mutant forms or wt EGFR could represent an advantage in terms of toxicity. It is not known whether the concentrations of siRNAs used in the present study will be achievable in vivo and in the clinic. The most appealing small molecule to test in a combination strategy with T790M-specific-siRNAs would be afatinib, the panHER irreversible inhibitor.

\section{Acknowledgments}

The project was funded by the National Cancer Plan Belgium (Grant NKP-29-011) and the Stichting Tegen Kanker, Belgium. This study was partly supported by the research fund of Boehringer Ingelheim $\mathrm{GmbH}$. Gang Chen was supported by the Chinese Scholarship Council (CSC) and the Vrije Universiteit Brussel (VUB) PhD scholarship.

\section{Appendix A. Supplementary data}

Supplementary data associated with this article can be found, in the online version, at http://dx.doi.org/10.1016/j.bbrc.2012.12.070.

\section{References}

[1] A. Jemal, F. Bray, M.M. Center, J. Ferlay, E. Ward, D. Forman, Global cancer statistics, CA Cancer J. Clin. 61 (2011) 69-90.

[2] D.W. Bell, T.J. Lynch, S.M. Haserlat, P.L. Harris, R.A. Okimoto, B.W. Brannigan, D.C. Sgroi, B. Muir, M.J. Riemenschneider, R.B. Iacona, A.D. Krebs, D.H. Johnson, G. Giaccone, R.S. Herbst, C. Manegold, M. Fukuoka, M.G. Kris, J. Baselga, J.S 
Ochs, D.A. Haber, Epidermal growth factor receptor mutations and gene amplification in non-small-cell lung cancer: molecular analysis of the IDEAL/ INTACT gefitinib trials, J. Clin. Oncol. 23 (2005) 8081-8092.

[3] R. Sordella, D.W. Bell, D.A. Haber, J. Settleman, Gefitinib-sensitizing EGFR mutations in lung cancer activate anti-apoptotic pathways, Science 305 (2004) 1163-1167.

[4] J.A. Engelman, T. Mukohara, K. Zejnullahu, E. Lifshits, A.M. Borras, C.M. Gale, G.N. Naumov, B.Y. Yeap, E. Jarrell, J. Sun, S. Tracy, X. Zhao, J.V. Heymach, B.E. Johnson, L.C. Cantley, P.A. Janne, Allelic dilution obscures detection of a biologically significant resistance mutation in EGFR-amplified lung cancer, J. Clin. Invest. 116 (2006) 2695-2706.

[5] C.H. Yun, K.E. Mengwasser, A.V. Toms, M.S. Woo, H. Greulich, K.K. Wong, M. Meyerson, M.J. Eck, The T790M mutation in EGFR kinase causes drug resistance by increasing the affinity for ATP, Proc. Natl. Acad. Sci. USA 105 (2008) 20702075.

[6] Y. Kim, J. Ko, Z. Cui, A. Abolhoda, J.S. Ahn, S.H. Ou, M.J. Ahn, K. Park, The EGFR T790M mutation in acquired resistance to an irreversible second-generation EGFR inhibitor, Mol. Cancer Ther. 11 (2012) 784-791.

[7] J.C.-H. Yang, M.H. Schuler, N. Yamamoto, K.J. O’Byrne, V. Hirsh, T. Mok, S.L. Geater, S.V. Orlov, C.-M. Tsai, M.J. Boyer, W.-C. Su, J. Bennouna, T. Kato, V. Gorbunova, K.H. Lee, R.N.H. Shah, D. Massey, R.M. Lorence, M. Shahidi, L.V Sequist, LUX-Lung 3: A randomized, open-label, phase III study of afatinib versus pemetrexed and cisplatin as first-line treatment for patients with advanced adenocarcinoma of the lung harboring EGFR-activating mutations, J. Clin. Oncol. 30 (Suppl.) (2012). abstr LBA7500.

[8] S.M. Rothenberg, J.A. Engelman, S. Le, D.J. Riese 2nd, D.A. Haber, J. Settleman, Modeling oncogene addiction using RNA interference, Proc. Natl. Acad. Sci. USA 105 (2008) 12480-12484.

[9] Z. Tang, R. Du, S. Jiang, C. Wu, D.S. Barkauskas, J. Richey, J. Molter, M. Lam, C. Flask, S. Gerson, A. Dowlati, L. Liu, Z. Lee, B. Halmos, Y. Wang, J.A. Kern, P.C. Ma, Dual MET-EGFR combinatorial inhibition against T790M-EGFR-mediated erlotinib-resistant lung cancer, Br. J. Cancer 99 (2008) 911-922.

[10] S. Yamanaka, Z. Gu, M. Sato, R. Fujisaki, K. Inomata, A. Sakurada, A. Inoue, T. Nukiwa, T. Kondo, A. Horii, SiRNA targeting against EGFR, a promising candidate for a novel therapeutic application to lung adenocarcinoma Pathobiology 75 (2008) 2-8.

[11] A. Reynolds, D. Leake, O. Boese, S. Scaringe, W.S. Marshall, A. Khvorova, Rational siRNA design for RNA interference, Nat. Biotechnol. 22 (2004) 326330.
[12] K. Ui-Tei, Y. Naito, F. Takahashi, T. Haraguchi, H. Ohki-Hamazaki, A. Juni, R. Ueda, K. Saigo, Guidelines for the selection of highly effective siRNA sequences for mammalian and chick RNA interference, Nucleic Acids Res. 32 (2004) 936948.

[13] B. Jagla, N. Aulner, P.D. Kelly, D. Song, A. Volchuk, A. Zatorski, D. Shum, T Mayer, D.A. De Angelis, O. Ouerfelli, U. Rutishauser, J.E. Rothman, Sequence characteristics of functional siRNAs, RNA 11 (2005) 864-872.

[14] G. Chen, P. Kronenberger, E. Teugels, I. Adaku Umelo, J. De Greve, Targeting the epidermal growth factor receptor in non-small cell lung cancer cells: the effect of combining RNA interference with tyrosine kinase inhibitors or cetuximab, BMC Med. 10 (2012) 28.

[15] G. Chen, P. Kronenberger, I.A. Umelo, E. Teugels, J. De Grève, Quantification of epidermal growth factor receptor T790M mutant transcripts in lung cancer cells by real-time reverse transcriptase-quantitative polymerase chain reaction, Anal. Biochem. 398 (2010) 266-268.

[16] G. Chen, P. Kronenberger, E. Teugels, J. De Grève, Influence of RT-qPCR primer position on EGFR interference efficacy in lung cancer cells, Biol. Proced. Online 13 (2011) 1.

[17] R. Koivusalo, E. Krausz, H. Helenius, S. Hietanen, Chemotherapy compounds in cervical cancer cells primed by reconstitution of p53 function after short interfering RNA-mediated degradation of human papillomavirus 18 E6 mRNA: opposite effect of siRNA in combination with different drugs, Mol. Pharmacol. 68 (2005) 372-382.

[18] T.C. Chou, Theoretical basis, experimental design, and computerized simulation of synergism and antagonism in drug combination studies, Pharmacol. Rev. 58 (2006) 621-681.

[19] L. Regales, Y. Gong, R. Shen, E. de Stanchina, I. Vivanco, A. Goel, J.A. Koutcher, M. Spassova, O. Ouerfelli, I.K. Mellinghoff, M.F. Zakowski, K.A. Politi, W. Pao, Dual targeting of EGFR can overcome a major drug resistance mutation in mouse models of EGFR mutant lung cancer, J. Clin. Invest. 119 (2009) 30003010.

[20] Y.Y. Janjigian, H.J. Groen, L. Horn, E.F. Smit, Y. Fu, F. Wang, M. Shahidi, L.J. Denis, W. Pao, V.A. Miller, Activity and tolerability of afatinib (BIBW 2992) and cetuximab in NSCLC patients with acquired resistance to erlotinib or gefitinib, J. Clin. Oncol. (Suppl.) (2011). Abstract No:7525^.

[21] J.M. Roda, T. Joshi, J.P. Butchar, J.W. McAlees, A. Lehman, S. Tridandapani, W.E. Carson 3rd, The activation of natural killer cell effector functions by cetuximab-coated, epidermal growth factor receptor positive tumor cells is enhanced by cytokines, Clin. Cancer Res. 13 (2007) 6419-6428. 